\title{
Risk factors for postoperative intracranial infections in patients with pituitary adenoma after endoscopic endonasal transsphenoidal surgery: pneumocephalus deserves further study
}

\author{
*Kang Guo, MD, ${ }^{1}$ Lijun Heng, MD, PhD, ${ }^{1}$ Haihong Zhang, MM, ${ }^{1}$ Lei Ma, MM, ${ }^{1}$ Hui Zhang, MS, ${ }^{2}$ and \\ Dong Jia, MD, PhD' \\ 1Department of Neurosurgery, Tangdu Hospital, Air Force Medical University, Xi'an, Shaanxi; ${ }^{2}$ State Key Laboratory of Genetic \\ Engineering and Ministry of Education, College of Life Sciences, Fudan University, Shanghai, China
}

\begin{abstract}
OBJECTIVE The authors sought to identify the relevance between pneumocephalus and postoperative intracranial infections, as well as bacteriological characteristics and risk factors for intracranial infections, in patients with pituitary adenomas after endoscopic endonasal transsphenoidal surgery.
\end{abstract}

METHODS In total, data from 251 consecutive patients with pituitary adenomas who underwent pure endoscopic endonasal transsphenoidal surgeries from 2014 to 2018 were reviewed for preoperative comorbidities, intraoperative techniques, and postoperative care.

RESULTS This retrospective study found 18 cases of postoperative pneumocephalus (7.17\%), 9 CNS infections (3.59\%), and 12 CSF leaks (4.78\%). Of the patients with pneumocephalus, 5 (27.8\%) had CNS infections. In patients with CNS infections, the culture results were positive in 7 cases and negative in 2 cases. The statistical analysis suggested that pneumocephalus (maximum bubble diameter of $\geq 1 \mathrm{~cm}$ ), diaphragmatic defects (intraoperative CSF leak, Kelly grade $\geq 1$ ), and a postoperative CSF leak are risk factors for postoperative CNS infections.

CONCLUSIONS In pituitary adenoma patients who underwent pure endoscopic endonasal transsphenoidal surgeries, intraoperative saddle reconstruction has a crucial role for patients with postoperative intracranial infections. Additionally, postoperative pneumocephalus plays an important role in predicting intracranial infections that must not be neglected. Therefore, neurosurgeons should pay close attention to the discovery of postoperative intracranial pneumocephalus because this factor is as important as a postoperative CSF leak. Pneumocephalus (maximum bubble diameter of $\geq 1 \mathrm{~cm}$ ), diaphragmatic defects (an intraoperative CSF leak, Kelly grade $\geq 1$ ), and a postoperative CSF leak were risk factors predictive of postoperative intracranial infections. In addition, it is essential that operative procedures be carefully performed to avoid diaphragmatic defects, to reduce exposure to the external environment, and to decrease patients' suffering . https://thejns.org/doi/abs/10.3171/2019.5.FOCUS19269

KEYWORDS pneumocephalus; intracranial infection; pituitary adenoma; cerebrospinal fluid leak; transsphenoidal surgery; diaphragmatic defects

$\mathrm{M}$ ODIFICATIONS and refinements of the endoscopic endonasal transsphenoidal approach have occurred since the 1960s, and this technique has become widely employed as a treatment for pituitary adenomas and other parasellar tumors because of its safety and effectiveness. ${ }^{17}$ Specifically, this approach provides a better choice for several specific anterior skull base lesions, such as suprasellar and clival tumors. ${ }^{16}$ In contrast to microscopic transsphenoidal and open transcranial approaches, the endoscopic endonasal transsphenoidal approach permits aggressive resection and superior outcomes via a narrow access corridor with minimal lesion left in the surrounding tissue..$^{8,12,13}$

However, the major concerns related to endoscopic endonasal transsphenoidal surgery include the risks of CSF rhinorrhea, intracranial infections, diabetes insipidus, pneumocephalus, and olfactory dysfunction. ${ }^{15}$ Destruction of the skull bone after endoscopic surgery always means

ABBREVIATIONS $\mathrm{BMI}=$ body mass index; NSF = nasal septal flap

SUBMITTED March 30, 2019. ACCEPTED May 20, 2019.

INCLUDE WHEN CITING DOI: 10.3171/2019.5.FOCUS19269.

${ }^{*}$ K.G. and L.H. contributed equally to this study and share first authorship. 
TABLE 1. Kelly grade of intraoperative cerebrospinal fluid leak

\begin{tabular}{cc}
\hline $\begin{array}{c}\text { Grade } \\
\text { of Leak }\end{array}$ & \multicolumn{1}{c}{ Description of Leak } \\
\hline Grade 0 & $\begin{array}{c}\text { Absence of cerebrospinal fluid leak, confirmed by the } \\
\text { Valsalva maneuver }\end{array}$ \\
\hline Grade 1 & $\begin{array}{c}\text { Small "weeping" leak, confirmed by the Valsalva maneuver, } \\
\text { without or with only a small diaphragmatic defect }\end{array}$ \\
\hline Grade 2 & $\begin{array}{c}\text { Moderate cerebrospinal fluid leak, with a clear diaphrag- } \\
\text { matic defect }\end{array}$ \\
\hline Grade 3 & $\begin{array}{c}\text { Large cerebrospinal fluid leak, typically created as part } \\
\text { of an extended transsphenoidal approach through the } \\
\text { supradiaphragmatic or clival dura for tumor access }\end{array}$ \\
\hline
\end{tabular}

Republished with permission of Congress of Neurosurgical Surgeons, Graded repair of cranial base defects and cerebrospinal fluid leaks in transsphenoidal surgery, Esposito et al., Neurosurgery 60 (4 Suppl 2), 2007.

that the cranial cavity is subjected to the outside environment. The lack of protection from the bone, dura mater, and blood-brain barrier and other factors increases the risk of intracranial infections. Therefore, following tumor removal, a series of measures should be implemented to repair surgical defects so as to avoid postoperative CSF leaks, intracranial infections, and pneumocephalus. The postoperative intracranial infection rate has not been clearly established; nevertheless, according to previous studies, the CSF rhinorrhea and intracranial infection rates range from $0.7 \%$ to $10 \%{ }^{4,9,14,22,23}$

Several studies have suggested that intracranial infection is closely associated with CSF rhinorrhea., ${ }^{4,22}$ However, no previous study definitively described the relevance between postoperative intracranial pneumocephalus and intracranial infections. This study aims to identify the relevance between pneumocephalus and postoperative meningitis and provide data to support this relevance. Although pneumocephalus after endoscopic endonasal transsphenoidal surgery is not uncommon, we hope that the data set in this study will cause neurosurgeons to pay more attention to this risk factor because it plays an important role in predicting intracranial infection such as postoperative CSF leak. In addition, meticulous performance and improvement of the details of the surgical procedure may decrease the occurrence of these complications. Essential operative procedures should be carefully considered to reduce the incidence of postoperative intracranial infection in patients undergoing nasal endoscopic surgery and to decrease patients' suffering.

\section{Methods \\ Data Source}

This retrospective study was reviewed and approved by the Institutional Review Board of Tangdu Hospital, Air Force Medical University. This retrospective study evaluated 251 consecutive pituitary adenoma patients who underwent a pure endoscopic endonasal transsphenoidal surgery performed by Dr. Jia between March 2014 and June 2018.

Detailed information was recorded for each patient, including age, sex, body mass index (BMI), clinical manifes- tations of the disease, comorbidities (including hypertension and diabetes), smoking and drinking habits, previous surgical procedures (endonasal procedures or craniotomies), presence of a concurrent infection that required antibiotics, duration of surgery ( $<4$ hours or $\geq 4$ hours), ${ }^{3}$ lumbar drain placement immediately after surgery, the presence of an intraoperative CSF leak according to the Kelly grading system $^{6}$ (Table 1), intraoperative blood loss, presence of a postoperative CSF leak, postoperative duration of diabetes insipidus, and postoperative pneumocephalus. In addition, all patients underwent postoperative CT and MRI examinations to assess surgical outcomes.

\section{Intraoperative Steps}

Two neurosurgeons (D.J. and assistant L.H.) performed all operations. The preoperative preparation included sterilization of the meatus using pledgets saturated with iodophor and contraction of the nasal mucosa using pledgets saturated with a mixed solution that contained $20 \mathrm{mg} / \mathrm{ml}$ of lidocaine $\mathrm{HCl}$ and $0.0125 \mathrm{mg} / \mathrm{ml}$ epinephrine $\mathrm{HCl}$. These pledgets were placed on the medial side of the turbinates through both nostrils to achieve mucosal decongestion and to decrease mucosal bleeding during the nasal phase of the surgery. ${ }^{2}$ A nasal septal flap (NSF) was recommended for all patients with large diaphragmatic defects.., 10

Following the preoperative preparation, the middle turbinate was lateralized to obtain a wider operating space, although it was not routinely resected. ${ }^{9}$ Then the NSF was harvested as a mucoperichondrial flap through the sphenoid sinus openings based on the posterior nasoseptal artery. The anterior sella was opened using a high-speed drill and enlarged using rongeurs. After the sphenoid sinus mucosa was removed, the optic nerve and carotid canal buttresses were visualized as landmarks. The floor of the sella was identified and opened, after which the dura mater was cut, and the tumors were resected using ring curettes and suction.

At the end of the procedure, the sella region was reconstructed carefully. When the Kelly grading score was $<2$ points, the defect was covered with a collagen sponge and synthetic dura (Duraform, Codman \& Shurtleff, Inc.), and the sphenoid sinus was filled with Gelfoam (Surgiflo, Ferrosan Medical Devices). When the Kelly grading score was $\geq 2$, the defect in the sella area was covered with one or two layers of thigh fat and muscle fascia using surgical glue..$^{19,21}$

The NSF was subsequently laid over the sellar base, and the wound was covered with Gelfoam. Finally, a Foley balloon catheter (10-12 Fr) was placed across the nasopharynx posterior to the nasal septum and filled with appropriate saline. ${ }^{20}$ An expansive sponge (Spiggle \& Theis Medizintechnik $\mathrm{GmbH}$ ) was also typically placed underneath the balloon. Ceftriaxone or cefminox was used for perioperative prophylaxis. Antibiotics were given $30 \mathrm{~min}$ utes before skin or mucosal incision and commonly used until 24 hours after operation. The expansive sponges and balloon were removed after 5-7 days. ${ }^{5}$

\section{Outcome Assessment}

Both the location and size of the pneumocephalus 

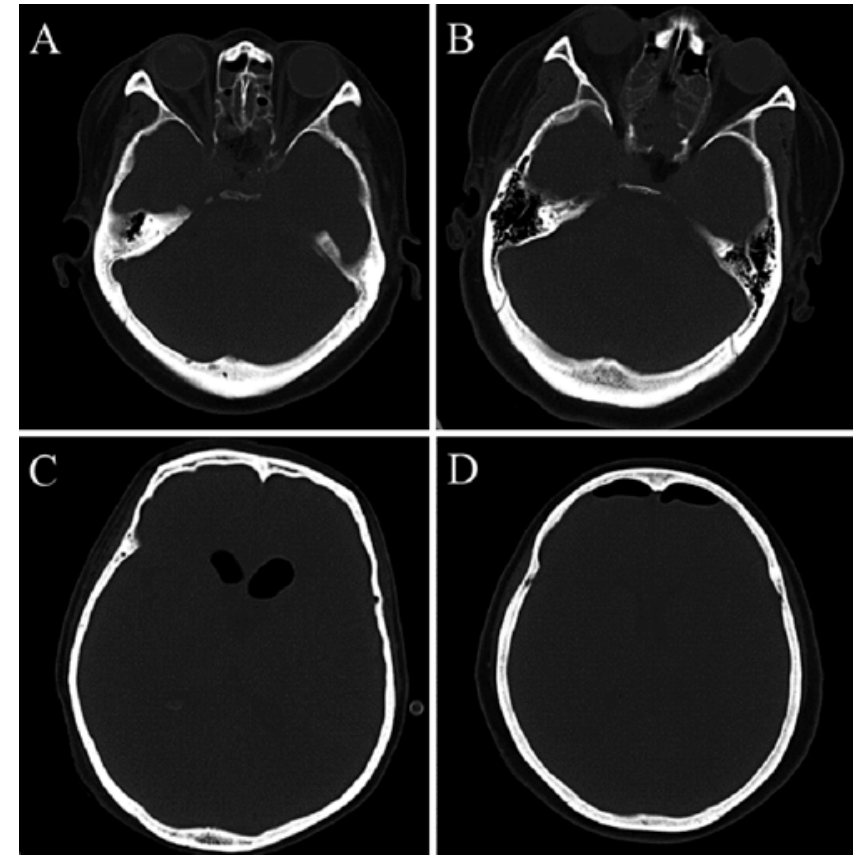

FIG. 1. CT scans of patients with postoperative pneumocephalus, showing examples of the 3 different pneumocephalus patterns based on anatomical location: normal brain (A), sellar region (B), intraventricular (C), and frontal (D).

were recorded for all patients. Postoperative CT and MRI scans obtained within 1-10 days were examined, and pneumocephalus was graded based on the location and size. A postoperative pneumocephalus shown 7-10 days on delayed scans was considered to be persistent pneumocephalus. Additionally, in this retrospective study, data on persistent pneumocephalus are used and collected as our key data to predict the risk of postoperative infection. The three different anatomical patterns (Fig. 1) were classified as frontal, intraventricular, and sellar region. The degree of pneumocephalus was characterized based on the following scale: 0 (none) and 1 (bubbles $\geq 1 \mathrm{~cm}$ in maximum diameter). The size of the pneumocephalus was established based on the largest diameter of bubbles. ${ }^{1}$ Drs. Haihong Zhang and Lei Ma were responsible for identifying the pneumocephalus if it was present. Postoperative meningitis was assessed for at least one month (no matter the end of admission).

In patients who were suspected to have an intracranial infection, CSF samples were collected via lumbar puncture and cultured. Data were collected from the CSF analysis and laboratory examinations, including a complete blood cell count, and all microorganisms were identified from blood samples and CSF cultures. Ceftriaxone or cefminox was used for conventional perioperative prophylaxis. ${ }^{3}$ The treatment of patients in whom intracranial infections developed following the operation depended on the drug sensitivity observed in the CSF cultures. Postoperative CSF rhinorrhea was confirmed by the presence of $\beta 2$-transferrin and/or glucose in the nasal fluid. ${ }^{14}$

The Centers for Disease Control and Prevention criteria were used to diagnose intracranial infection ${ }^{7,18}$ (Table 2). Detailed information was recorded for patients with
TABLE 2. Diagnosis of an intracranial infection

\begin{tabular}{l}
\hline Definitive diagnosis of an infection \\
Infection definitively diagnosed based on CSF cultures that were \\
positive for a likely pathogen or a positive Gram stain result* \\
Diagnosis of a potential infection \\
\hline 1) Fever (temperature $\geq 38^{\circ} \mathrm{C}$ ), headache, stiff neck, meningeal \\
signs, cranial nerve signs, \&/or irritability w/ no other recog- \\
nized cause \\
2) Increased white blood cell count, increased protein level, \&/or \\
decreased glucose level in the CSF, \&/or a positive blood \\
culture result \\
3) A full course of antibiotics was administered
\end{tabular}

${ }^{*}$ Criteria are based on those used by the Centers for Disease Control and Prevention. ${ }^{7,18}$

intracranial infections, including the clinical presentation, culture and chemical analysis of the CSF, treatment and outcome. The surgical records were assessed for intraoperative CSF leakage, duration of surgery $(<4$ hours or $\geq 4$ hours), and intraoperative blood loss.

Lumbar drainage and lumbar puncture are not routinely used after surgery. However, when a lumbar drain was placed, it remained for no more than 3 days, at a maximum rate of $5 \mathrm{ml} /$ hour in patients with a high-flow postoperative CSF leak, while patients with low-flow CSF leaks are recommended to undergo a lumbar puncture to keep the wound dry; to sit in a bench-like position; or to raise their head to a height of $15^{\circ}-30^{\circ}$ in a semirecumbent position for 3 days after surgery to avoid placement of a lumbar drain.

\section{Statistical Analysis}

The data are expressed as the mean \pm SD for the continuous variables and as numbers and percentages for the categorical variables. The chi-square test and Fisher's exact test were used for comparisons, when appropriate. A multivariate logistic regression model was used to select factors significantly associated with intracranial infections. All variables were used to compare the two groups of patients: patients with and patients without postoperative intracranial infections. Analyses were performed using SPSS software version 19 (IBM Corp.), and $\mathrm{p}$ values of 0.05 or less were considered statistically significant.

\section{Results}

\section{Patient Demographics}

Of 251 patients studied, 18 (7.17\%) had postoperative pneumocephalus, 9 (3.59\%) had CNS infections, and 12 (4.78\%) had CSF leaks. In pneumocephalus patients, 5 (27.8\%) had CNS infections. In 9 patients with CNS infectious, the culture results were positive in 7 cases (Table 3; cases 1-7) and negative in 2 cases (Table 3; cases 8 and 9). A total of 116 women (46.2\%) and 135 men $(53.8 \%)$ underwent surgery. The average age $( \pm \mathrm{SD})$ of patients who underwent surgery was $47.65 \pm 12.36$ years. Six patients (2.39\%) had a history of endonasal surgery or craniotomy. The duration of surgery was $\geq 4$ hours for 39 procedures (16.1\%). Overall, 12 patients (4.78\%) had a postoperative 




CSF leak, 3 patients required treatment with a lumbar drain, and 2 required endoscopic repair. Based on the degree of the intraoperative CSF leak classification (Kelly grade classification), $41(16.33 \%)$ of the 251 procedures were found to have a recognized intraoperative CSF leak, including 17 grade 1 (6.77\%), 14 grade $2(5.58 \%)$, and 10 grade $3(3.98 \%)$ leaks. In addition, in 9 postinfectious cases, 5 patients $(27.8 \%)$ were identified as having postpneumocephalus air $(\mathrm{p}<0.01)$ and 6 patients $(66.67 \%)$ as having postoperative CSF leaks ( $\mathrm{p}<0.001)$.

\section{Pneumocephalus}

Of the 251 procedures examined, postoperative persistent or delayed pneumocephalus was found in 18 patients (7.17\%), including 13 with frontal pneumocephalus, 3 with intraventricular pneumocephalus, and 2 with sellar pneumocephalus (Fig. 1). Of 9 patients with an infection, $5(55.6 \%)$ developed postoperative pneumocephalus $(\mathrm{p}<$ $0.01)$.

\section{Incidence of Infection and Risk Factors for Infection}

In this study, 9 of 251 patients were found to have intracranial infections, which puts the infection rate at $3.59 \%$. Seven patients had positive culture results (Table 3; cases 1-7) and 2 patients had negative results (Table 3; cases $8-9)$. The mean age of the patients who developed an intracranial infection after surgery was $47.65 \pm 12.36$ years. Detailed information concerning the patients with infections is presented in Table 3. Among the patients with intracranial infections, methicillin-resistant Staphylococcus aureus was cultured from the CSF obtained from 2 patients. Klebsiella pneumoniae, Acinetobacter baumannii, Enterobacter cloacae, and gram-positive Staphylococcus epidermidis and Streptococcus pneumoniae were each identified in a single patient.

According to the univariate analysis (Table 4), pneumocephalus (a maximum bubble diameter of $\geq 1 \mathrm{~cm}$ ), postoperative diabetes insipidus, diaphragmatic defects (intraoperative CSF leak, Kelly grade $\geq 1$ ), and postoperative CSF leakage are risk factors for postoperative intracranial infections. Male sex, age over 60 years, abnormal BMI, history of surgery (craniotomy or endonasal surgery), and diagnosis of hypertension or diabetes are not risk factors. Additionally, the multivariate logistic regression model (Table 5) suggested that pneumocephalus (a maximum bubble diameter of $\geq 1 \mathrm{~cm}$ ), diaphragmatic defects (intraoperative CSF leak, Kelly grade $\geq 1$ ), and postoperative CSF leak are risk factors for postoperative intracranial infections.

\section{Tumor Pathology}

The pathology included nonfunctional pituitary adenoma $(n=210)$, growth hormone-secreting pituitary adenoma $(n=37)$, and prolactin-secreting pituitary adenoma $(n=4)$.

\section{Discussion}

Improvements in the endoscopic endonasal transsphenoidal approach have allowed this technique to achieve a 
TABLE 4. Risk factors for intracranial infections after endoscopic endonasal transsphenoidal surgery based on the results of a univariate analysis

\begin{tabular}{lccc}
\hline & \multicolumn{2}{c}{ No. of Cases (\%) } & \\
\cline { 2 - 3 } \multicolumn{1}{c}{ Risk Factor } & $\begin{array}{c}\text { Noninfected Pts } \\
(\mathrm{n}=242)\end{array}$ & $\begin{array}{c}\text { Infected Pts } \\
(\mathrm{n}=9)\end{array}$ & p Value \\
\hline Male sex & $129(53.3)$ & $6(66.7)$ & 0.511 \\
\hline Age >60 yrs & $45(18.6)$ & $2(22.2)$ & 0.677 \\
\hline Abnormal BMI & $86(35.5)$ & $3(33.3)$ & 0.892 \\
\hline $\begin{array}{l}\text { History of endonasal surgery } \\
\quad \text { or craniotomy }\end{array}$ & $6(2.48)$ & $0(0)$ & 0.633 \\
\hline Duration of surgery $\geq 4$ hrs & $39(16.1)$ & $3(3.33)$ & 0.177 \\
\hline Hypertension & $33(13.6)$ & $1(1.11)$ & 0.828 \\
\hline Diabetes & $17(7.02)$ & $0(0)$ & 0.410 \\
\hline Intraop CSF leak & $34(14.0)$ & $7(77.8)$ & $<0.001$ \\
\hline Postop diabetes insipidus & $4(1.65)$ & $5(55.6)$ & $<0.001$ \\
\hline Pneumocephalus $(\geq 1 \mathrm{~cm})$ & $13(5.37)$ & $5(55.6)$ & $<0.001$ \\
\hline Postop CSF leak & $6(2.48)$ & $6(66.7)$ & $<0.001$ \\
\hline
\end{tabular}

Pts $=$ patients.

higher rate of total resection, provide faster recovery, and avoid disturbances and destruction of the surrounding brain structures and important nerve vascular tissue. A previous study has indicated that endoscopic methods allow for a greater rate of total resection and improved postoperative visual outcomes compared with a craniotomy. ${ }^{12}$ In this retrospective study, 18 cases $(7.17 \%)$ of postoperative pneumocephalus, 9 cases of CNS infection (3.59\%), and 12 cases of CSF leaks (4.78\%) were identified. Overall, the incidence of intracranial infection after traditional transsphenoidal surgery ranges from $0.7 \%$ to $10 \%, 4,9,14,22,23$ which is comparable to the $3.59 \%$ incidence identified in this study.

\section{Risk Factors for Intracranial Infection}

As our multivariate logistic regression analysis suggested, diaphragmatic defects (intraoperative CSF leak, Kelly grade $\geq 1$ ), pneumocephalus (maximum bubble diameter of $\geq 1 \mathrm{~cm}$ ), and a postoperative CSF leak are risk factors for a postoperative intracranial infection. The results of this retrospective study have proven the relevance between pneumocephalus and postoperative intracranial infections. Although pneumocephalus after endoscopic endonasal transsphenoidal surgery is not uncommon, the data set in this study will cause neurosurgeons to pay more attention to this risk factor because it plays as important a role in predicting intracranial infection as postoperative CSF leak. In patients with pneumocephalus (maximum bubble diameter of $\geq 1 \mathrm{~cm}$ ), large diaphragmatic defects, and intraoperative CSF leaks, the brain tissue easily communicates with the external environment to increase the risk of intracranial infection. As pneumocephalus (maximum bubble diameter of $\geq 1 \mathrm{~cm}$ ) is a risk factor, surgical procedures should be performed carefully to protect the nature of the barrier, to avoid intraoperative CSF leaks, and to reduce the incidence of postoperative intracranial infections.
TABLE 5. Risk factors for intracranial infections after endoscopic endonasal transsphenoidal surgery based on the results of a multivariate logistic regression analysis

\begin{tabular}{lcc}
\hline \multicolumn{1}{c}{ Variable } & $p$ Value & Odds Ratio $(95 \% \mathrm{Cl})$ \\
\hline $\begin{array}{l}\text { Diaphragmatic defect (Kelly } \\
\text { grade } \geq 1)\end{array}$ & 0.034 & $7.868(1.172-52.822)$ \\
\hline Pneumocephalus & 0.030 & $8.324(1.231-56.270)$ \\
\hline Postop CSF leak & 0.001 & $22.278(3.435-144.474)$ \\
\hline
\end{tabular}

The presence of postoperative pneumocephalus indicates there are clear links between the cranial cavity and the external environment, a link that is shared with postoperative CSF leaks. The difference between postoperative pneumocephalus and a postoperative CSF leak is that the pneumocephalus originates from a contaminated external source and goes into the sterile cranial cavity, whereas the CSF leak occurs from the ventricles of the brain to the external environment. Thus, in pneumocephalus patient bacteria may more easily enter the cranial cavity from the external environment through a potential gap and cause a postoperative infection. Compared with a frontal pneumocephalus, an intraventricular pneumocephalus indicates an infiltration of gas deep into the brain, which results in a postoperative intracranial infection. The observation that pneumocephalus (maximum bubble diameter of $\geq 1 \mathrm{~cm}$ ) is a risk factor highlights this point. It is worth mentioning that the size of the pneumocephalus was established based on the largest diameter because of the irregular shape of the postoperative pneumocephalus, indicating that the volume of the intracranial pneumocephalus cannot be measured accurately. At this point, sealing the saddle area is particularly important and may decrease the risk of a patient developing a postoperative intracranial infection. The use of medical glue may increase the sealing performance. However, further studies are required to determine whether this glue will reduce the risk of postoperative intracranial infections and CSF leakage.

We analyzed the infection rate and its associated pre-, intra-, and postoperative risk factors. The results confirm that intraoperative saddle reconstruction plays a crucial role for patients with postoperative intracranial infections. Operative procedures should be carefully performed to avoid large diaphragmatic or dural defects and to reduce the possibility of the cranial cavity becoming exposed to the external environment, which suggests that postoperative pneumocephalus deserves further study. Additionally, postoperative pneumocephalus might be a risk factor that neurosurgeons easily ignore in clinical practice. The diaphragmatic defect is an important factor that predicts the presence of postoperative intracranial infections, particularly in patients with pituitary adenomas. However, other tumors, such as craniopharyngiomas, chordomas, or meningiomas, may not be completely comparable to pituitary adenomas because they are more invasive and have a firmer texture, which might have breached the diaphragm. In other words, the diaphragm might have been previously infiltrated by these tumors prior to the operation, thereby increasing the likelihood of communication between the 
external environment and the cranial cavity and leading to postoperative intracranial infections.

Finally, the NSF was used in each patient with large dural or diaphragmatic defects. Several recent studies have reported an association between olfactory problems and the use of this flap. In our experience, the upper edge of the nasal septal mucosal flap should not be placed too close to the superior turbinate. The use of monopolar electrocoagulation around the olfactory mucosa of the superior turbinate may damage the olfactory fila, resulting in olfactory dysfunction. The use of a cold knife to perform the superior incision may reduce tissue damage and provide better olfactory outcomes. ${ }^{11}$

\section{Several Techniques to Prevent Infection}

Several techniques may be used after the tumor is resected to decrease the risk of postoperative pneumocephalus and infection. First, a sufficient amount of saline should be given to slowly wash the saddle area and expel the intracranial gas. Second, during saddle reconstruction, the cranial defect may be covered with a collagen sponge and synthetic dura, and the sphenoid sinus filled with Gelfoam; using an autologous fat graft in place of an artificial dural substitute could help in preventing any persistent communication between the extracranial and intracranial cavity after surgery, especially in patients with a CSF leak. Then, patients can maintain themselves in the bench-like position for 3 days after surgery or raise their heads to a height of $15^{\circ}-30^{\circ}$ in a semirecumbent position. Moreover, sneezing and sniffling should be avoided after surgery because they can easily increase intracranial pressure and thereby cause persistent communication between the extracranial and intracranial cavity. In addition, overdrainage should be avoided in patients who have a lumbar drain, because it could cause intracranial negative pressure and result in persistent pneumocephalus. Last, oxygen is recommended via mask and nasal catheter, and it is better for the nasal catheter to be placed in the patient's mouth. The ventilator and endotracheal tube are routinely removed after the patient wakes up.

\section{Study Limitations}

The limitations of the present study include the bias associated with a retrospective analysis. The sample size of this retrospective study was relatively small. In addition, because of the irregular shape of the postoperative pneumocephalus, the size of the pneumocephalus was established based on the largest diameter. Therefore, the volume of the intracranial pneumocephalus could not be accurately measured. Unfortunately, the lumbar drainage data were missing in some patients, especially in patients without a postoperative CSF leak and pneumocephalus.

\section{Conclusions}

Using an endoscopic endonasal transsphenoidal approach, intraoperative saddle reconstruction has a crucial role for patients with postoperative intracranial infections. In addition, neurosurgeons should pay close attention when finding postoperative intracranial pneumocephalus so that they can manage the situation. Pneumocephalus (maximum bubble diameter of $\geq 1 \mathrm{~cm}$ ), diaphragmatic defects (an intraoperative CSF leak, Kelly grade $\geq 1$ ), and postoperative CSF leaks are risk factors for a postoperative intracranial infection. It is essential that operative procedures be carefully performed to avoid diaphragmatic defects, to reduce exposure to the external environment, and to decrease patients' suffering.

\section{References}

1. Banu MA, Szentirmai O, Mascarenhas L, Salek AA, Anand VK, Schwartz TH: Pneumocephalus patterns following endonasal endoscopic skull base surgery as predictors of postoperative CSF leaks. J Neurosurg 121:961-975, 2014

2. Berker M, Hazer DB, Yücel T, Gürlek A, Cila A, Aldur M, et al: Complications of endoscopic surgery of the pituitary adenomas: analysis of 570 patients and review of the literature. Pituitary 15:288-300, 2012

3. Dellinger EP, Gross PA, Barrett TL, Krause PJ, Martone WJ, McGowan JE Jr, et al: Quality standard for antimicrobial prophylaxis in surgical procedures. Clin Infect Dis 18:422427, 1994

4. Dumont AS, Nemergut EC II, Jane JA Jr, Laws ER Jr: Postoperative care following pituitary surgery. J Intensive Care Med 20:127-140, 2005

5. El-Sayed IH, Roediger FC, Goldberg AN, Parsa AT, McDermott MW: Endoscopic reconstruction of skull base defects with the nasal septal flap. Skull Base 18:385-394, 2008

6. Esposito F, Dusick JR, Fatemi N, Kelly DF: Graded repair of cranial base defects and cerebrospinal fluid leaks in transsphenoidal surgery. Neurosurgery 60 (4 Suppl 2):295-304, 2007

7. Garner JS, Jarvis WR, Emori TG, Horan TC, Hughes JM: CDC definitions for nosocomial infections, 1988. Am J Infect Control 16:128-140, 1988

8. Hardy J: Transsphenoidal hypophysectomy. 1971. J Neurosurg 107:458-471, 2007

9. Ivan ME, Iorgulescu JB, El-Sayed I, McDermott MW, Parsa AT, Pletcher SD, et al: Risk factors for postoperative cerebrospinal fluid leak and meningitis after expanded endoscopic endonasal surgery. J Clin Neurosci 22:48-54, 2015

10. Kassam AB, Prevedello DM, Carrau RL, Snyderman CH, Thomas A, Gardner P, et al: Endoscopic endonasal skull base surgery: analysis of complications in the authors' initial 800 patients. J Neurosurg 114:1544-1568, 2011

11. Kim SW, Park KB, Khalmuratova R, Lee HK, Jeon SY, Kim DW: Clinical and histologic studies of olfactory outcomes after nasoseptal flap harvesting. Laryngoscope 123:16021606,2013

12. Komotar RJ, Starke RM, Raper DM, Anand VK, Schwartz TH: Endoscopic endonasal compared with microscopic transsphenoidal and open transcranial resection of craniopharyngiomas. World Neurosurg 77:329-341, 2012

13. Komotar RJ, Starke RM, Raper DM, Anand VK, Schwartz TH: Endoscopic endonasal compared with microscopic transsphenoidal and open transcranial resection of giant pituitary adenomas. Pituitary 15:150-159, 2012

14. Kono Y, Prevedello DM, Snyderman CH, Gardner PA, Kassam AB, Carrau RL, et al: One thousand endoscopic skull base surgical procedures demystifying the infection potential: incidence and description of postoperative meningitis and brain abscesses. Infect Control Hosp Epidemiol 32:7783,2011

15. Lai LT, Trooboff S, Morgan MK, Harvey RJ: The risk of meningitis following expanded endoscopic endonasal skull base surgery: a systematic review. J Neurol Surg B Skull Base 75:18-26, 2014

16. Laufer I, Anand VK, Schwartz TH: Endoscopic, endonasal 
extended transsphenoidal, transplanum transtuberculum approach for resection of suprasellar lesions. J Neurosurg 106:400-406, 2007

17. Liu JK, Das K, Weiss MH, Laws ER Jr, Couldwell WT: The history and evolution of transsphenoidal surgery. J Neurosurg 95:1083-1096, 2001

18. Mangram AJ, Horan TC, Pearson ML, Silver LC, Jarvis WR: Guideline for prevention of surgical site infection, 1999. Am J Infect Control 27:96-134, 1999

19. Patel KS, Komotar RJ, Szentirmai O, Moussazadeh N, Raper DM, Starke RM, et al: Case-specific protocol to reduce cerebrospinal fluid leakage after endonasal endoscopic surgery. J Neurosurg 119:661-668, 2013

20. Snyderman C, Kassam A, Carrau R, Mintz A, Gardner P, Prevedello DM: Acquisition of surgical skills for endonasal skull base surgery: a training program. Laryngoscope 117:699-705, 2007

21. Snyderman CH, Kassam AB, Carrau R, Mintz A: Endoscopic reconstruction of cranial base defects following endonasal skull base surgery. Skull Base 17:73-78, 2007

22. van Aken MO, de Marie S, van der Lely AJ, Singh R, van den Berge JH, Poublon RML, et al: Risk factors for meningitis after transsphenoidal surgery. Clin Infect Dis 25:852-856, 1997

23. van Aken MO, Feelders RA, de Marie S, van de Berge JH, Dallenga AH, Delwel EJ, et al: Cerebrospinal fluid leak- age during transsphenoidal surgery: postoperative external lumbar drainage reduces the risk for meningitis. Pituitary 7:89-93, 2004

\section{Disclosures}

The authors report no conflict of interest concerning the materials or methods used in this study or the findings specified in this paper.

\section{Author Contributions}

Conception and design: Jia, Guo, Heng. Acquisition of data: Haihong Zhang, Ma. Analysis and interpretation of data: Hui Zhang. Drafting the article: Guo. Critically revising the article: Guo. Reviewed submitted version of manuscript: all authors. Approved the final version of the manuscript on behalf of all authors: Jia. Statistical analysis: Hui Zhang. Administrative/technical/material support: Heng. Study supervision: Jia.

\section{Correspondence}

Dong Jia: Tangdu Hospital, Air Force Medical University, Shaanxi, China.jiadong69@163.com. 